\title{
Evolution of Abundance Gradients along the Galactic Disk
}

\author{
J. L. Hou
}

Shanghai Astronomical Observatory, Shanghai, 200030, P. R. China

Nikos Prantzos \& Samuel Boissier

Institut d'Astrophysique de Paris, 75014 Paris, France

\begin{abstract}
A detailed investigation of the abundance gradients and their evolution along the Galactic disk has recently appeared (Hou, J. L., Prantzos, N., \& Boissier, S. 2000, A\&A, in press; astro-ph/0007164). A chemical evolution model of S. Boissier \& N. Pranzos (1999, MNRAS, 307,857 ) was quite successful in reproducing the main observational constraints both in the solar neighborhood and the entire Milky Way disk. Studied elements include $\mathrm{He}, \mathrm{C}, \mathrm{N}, \mathrm{O}, \mathrm{Ne}, \mathrm{Mg}, \mathrm{Al}, \mathrm{Si}, \mathrm{S}, \mathrm{Ar}$ and $\mathrm{Fe}$. We use metallicity dependent yields for massive stars with and without mass loss. We find that most observed abundance profiles are correctly reproduced by massive star yields, but $C$ and $N$ require supplementary sources. We argue that massive, mass losing stars can totally account for the abundance profile of $C$, while intermediate mass stars are the main source of $N$. We also find that the adopted "inside-out" formation scheme for the Milky Way disk produces abundance profiles steeper in the past. Using current data on planetary nebulae of type I, II, and III, on $N, N e, S, A r$ as observational constraints for gradient evolution, we find that it is difficult to conclude whether the gradient steepens or flattens with time. However, for a given interval of Galactic age, our model predicts that the corresponding abundance scatter is smaller in the inner disk than in the outer regions.
\end{abstract}

